\title{
ON A NEW ABSOLUTE SUMMABILITY METHOD
}

\section{W.T. SULAIMAN}

Department of Mathematics

College of Science

University of Qatar

P.O. Box 2713

Doha, QATAR

(Received July 1993 and in revised form March 21, 1997)

ABSTRACT. A theorem concerning some new absolute summability method is proved. Many other results, some of them known, are deduced.

KEY WORDS AND PHRASES: Absolute summability.

1991 AMS SUBJECT CLASSIFICATION CODES: 40C.

\section{INTRODUCTION}

Let $\Sigma a_{n}$ be an infinite series with partial sums $s_{n}$ Let $\sigma_{n}^{\delta}$ and $\eta_{n}^{\delta}$ denote the $n$th Cesàro mean of order $\delta(\delta>-1)$ of the sequences $\left\{s_{n}\right\}$ and $\left\{n a_{n}\right\}$ respectively. The series $\Sigma a_{n}$ is said to be summable $|C, \delta|_{k}, k \geq 1$, if

$$
\sum_{n=1}^{\infty} n^{k-1}\left|\sigma_{n}^{\delta}-\sigma_{n-1}^{\delta}\right|^{k}<\infty
$$

or equivalently

$$
\sum_{n=1}^{\infty} n^{-1}\left|\eta_{n}^{\delta}\right|^{k}<\infty
$$

Let $\left\{p_{n}\right\}$ be a sequence of positive numbers such that

$$
P_{n}=\sum_{v=0}^{n} p_{v} \rightarrow \infty \quad \text { as } n \rightarrow \infty\left(P_{-\imath}=p_{-\imath}=0, i \geq 1\right)
$$

The series $\Sigma a_{n}$ is said to be summable $\left|\bar{N}, p_{n}\right|_{k}, k \geq 1$, if (Bor [1]),

$$
\sum_{n=1}^{\infty}\left(P_{n} / p_{n}\right)^{k-1}\left|t_{n}-t_{n-1}\right|^{k}<\infty \text {, }
$$

where

$$
t_{n}=P_{n}^{-1} \sum_{v=0}^{n} p_{v} s_{v}
$$

The series $\Sigma a_{n}$ is said to be summable $\left|R, p_{n}\right|_{k}, k \geq 1$, if Bor [2],

$$
\sum_{n=1}^{\infty} n^{k-1}\left|t_{n}-t_{n-1}\right|^{k}<\infty
$$


If we take $p_{n}=1$, each of the two summabilities $\left|\bar{N}, p_{n}\right|_{k}$ and $\left|R, p_{n}\right|_{k}$ is the same as $|C, 1|_{k}$ summabililty Let $\left\{\phi_{n}\right\}$ be any sequence of positive numbers. The series $\Sigma a_{n}$ is said to be summable $\left|\bar{N}, p_{n}, \phi_{n}\right|_{k}, k \geq 1$, if (Sulaiman [3]),

$$
\sum_{n=1}^{\infty} \phi_{n}^{k-1}\left|t_{n}-t_{n-1}\right|^{k}<\infty
$$

It is clear that

$$
\left|\bar{N}, p_{n}, n\right|_{k}=\left|R, p_{n}\right|_{k},\left|\bar{N}, p_{n}, P_{n} / p_{n}\right|_{k}=\left|\bar{N}, p_{n}\right|_{k},\left|\bar{N}, p_{n}, 1\right|_{1}=\left|\bar{N}, p_{n}\right| \text {, and }|\bar{N}, 1, n|_{k}=|C, 1|_{k} \text {. }
$$

We assume $\left\{\alpha_{n}\right\},\left\{\beta_{n}\right\}$ and $\left\{q_{n}\right\}$ be sequences of positive numbers such that

$$
Q_{n}=\sum_{v=0}^{n} q_{v} \rightarrow \infty
$$

We prove the following.

THEOREM 1. Let $t_{n}$ denote the $\left(\bar{N}, p_{n}\right)$-mean of the series $\Sigma a_{n}$ and write $T_{n}=\beta_{n}^{1-1 / k} \Delta t_{n-1}$. If

$$
\begin{aligned}
& \sum_{n=v+1}^{\infty} \frac{\alpha_{n}^{k-1} q_{n}^{k}}{Q_{n}^{k} Q_{n-1}}=O\left\{\frac{\alpha_{v}^{k-1} q_{v}^{k-1}}{Q_{v}^{k}}\right\}, \\
& \sum_{n=1}^{\infty}\left(\frac{\alpha_{n}}{\beta_{n}}\right)^{k-1}\left(\frac{P_{n}}{p_{n}}\right)^{k}\left(\frac{q_{n}}{Q_{n}}\right)^{k}\left|\epsilon_{n}\right|^{k}\left|T_{n}\right|^{k}<\infty, \\
& \sum_{n=1}^{\infty}\left(\frac{\alpha_{n}}{\beta_{n}}\right)^{k-1}\left|\epsilon_{n}\right|^{k}\left|T_{n}\right|^{k}<\infty
\end{aligned}
$$

and

$$
\sum_{n=1}^{\infty}\left(\frac{\alpha_{n}}{\beta_{n}}\right)^{k-1}\left(\frac{P_{n-1}}{p_{n}}\right)^{k}\left|\Delta \epsilon_{n}\right|^{k}\left|T_{n}\right|^{k}<\infty,
$$

then the series $\Sigma a_{n} \epsilon_{n}$ is summable $\left|\bar{N}, q_{n}, \alpha_{n}\right|_{k}, k \geq 1$.

\section{PROOF OF THEOREM 1}

Let $\tau_{n}$ be the $\left(\bar{N}, q_{n}\right)$-mean of the series $\Sigma a_{n} \epsilon_{n}$. Then

$$
\begin{aligned}
\Delta \tau_{n-1}= & -\frac{q_{n}}{Q_{n} Q_{n-1}} \sum_{v=1}^{n} Q_{v-1} a_{v} \epsilon_{v} \\
= & -\frac{q_{n}}{Q_{n} Q_{n-1}}\left\{\sum_{v=1}^{n-1} \sum_{r=1}^{n} P_{r-1} a_{r} \Delta\left(P_{v-1}^{-1} Q_{v-1} \epsilon_{v}\right)+\left(\sum_{r=1}^{n} P_{r-1} a_{r}\right) P_{n-1}^{-1} Q_{n-1} \epsilon_{n}\right\} \\
= & -\frac{q_{n}}{Q_{n} Q_{n-1}} \sum_{v=1}^{n-1}\left(\frac{P_{v} P_{v-1}}{p_{v}} \beta_{v}^{1 / k-1} T_{v}\right)\left(-q_{v} P_{v-1}^{-1} \epsilon_{v}+\frac{p_{v}}{P_{v} P_{v-1}} Q_{v} \epsilon_{v}+Q_{v} P_{v}^{-1} \Delta \epsilon_{v}\right) \\
& +\left(\frac{P_{n}}{p_{n}}\right)\left(\frac{q_{n}}{Q_{n}}\right) \epsilon_{n} \beta_{n}^{1 / k-1} T_{n} \\
= & -\frac{q_{n}}{Q_{n} Q_{n-1}} \sum_{v=1}^{n-1}\left(-\frac{P_{v}}{p_{v}} q_{v} \epsilon_{v} \beta_{v}^{1 / k-1} T_{v}+Q_{v} \epsilon_{v} \beta_{v}^{1 / k-1} T_{v}+\frac{P_{v-1}}{p_{v}} Q_{v} \Delta \epsilon_{v} \beta_{v}^{1 / k-1} T_{v}\right) \\
& \quad+\left(\frac{P_{n}}{p_{n}}\right)\left(\frac{q_{n}}{Q_{n}}\right) \epsilon_{n} \beta_{n}^{1 / k-1} T_{n} \\
= & T_{n, 1}+T_{n, 2}+T_{n, 3}+T_{n, 4}, \text { say. }
\end{aligned}
$$

To prove the theorem, by Minkowski's inequality, it is sufficient to show that 


$$
\sum_{n=1}^{\infty} \alpha_{n}^{k-1}\left|T_{n, r}\right|^{k}<\infty, \quad r=1,2,3,4
$$

Applying Hölder's inequality, with indices $k$ and $k^{\prime}$, where $\frac{1}{k}+\frac{1}{k^{\prime}}=1$, we have

$$
\begin{aligned}
\sum_{n=2}^{m+1} \alpha_{n}^{k-1}\left|T_{n, 1}\right|^{k} & =\sum_{n=2}^{m+1} \alpha_{n}^{k-1}\left(\frac{q_{n}}{Q_{n} Q_{n-1}}\right)^{k}\left|\sum_{v=1}^{n-1}-\frac{P_{v}}{p_{v}} q_{v} \epsilon_{v} \beta_{v}^{1 / k-1} T_{v}\right|^{k} \\
& \leq \sum_{n=2}^{m+1} \alpha_{n}^{k-1}\left(\frac{q_{n}}{Q_{n}}\right)^{k} Q_{n-1}^{-1} \sum_{v=1}^{n-1}\left(\frac{P_{v}}{p_{v}}\right)^{k} q_{v}\left|\epsilon_{v}\right|^{k} \beta_{v}^{1-k}\left|T_{v}\right|^{k}\left\{\frac{1}{Q_{n-1}} \sum_{v=1}^{n-1} q_{v}\right\}^{k-1} \\
& \leq 0(1) \sum_{v=1}^{m}\left(\frac{P_{v}}{p_{v}}\right)^{k} q_{v}\left|\epsilon_{v}\right|^{k} \beta_{v}^{1-k}\left|T_{v}\right|^{k} \sum_{n=v+1}^{m+1} \alpha_{n}^{k-1}\left(\frac{q_{n}}{Q_{n}}\right)^{k} Q_{n-1}^{-1} \\
& \leq 0(1) \sum_{v=1}^{m}\left(\frac{\alpha_{v}}{\beta_{v}}\right)^{k-1}\left(\frac{P_{v}}{p_{v}}\right)^{k}\left(\frac{q_{v}}{Q_{v}}\right)^{k}\left|\epsilon_{v}\right|^{k}\left|T_{v}\right|^{k}
\end{aligned}
$$

$$
\begin{aligned}
\sum_{n=2}^{m+1} \alpha_{n}^{k-1}\left|T_{n, 2}\right|^{k} & =\sum_{n=2}^{m+1} \alpha_{n}^{k-1}\left(\frac{q_{n}}{Q_{n} Q_{n-1}}\right)^{k}\left|\sum_{v=1}^{n-1} \frac{Q_{v}}{q_{v}} q_{v} \epsilon_{v} \beta_{v}^{1 / k-1} T_{v}\right|^{k} \\
& \leq \sum_{n=2}^{m+1} \alpha_{n}^{k-1}\left(\frac{q_{n}}{Q_{n}}\right)^{k} Q_{n-1}^{-1} \sum_{v=1}^{n-1}\left(\frac{Q_{v}}{q_{v}}\right)^{k} q_{v}\left|\epsilon_{v}\right|^{k} \beta_{v}^{1-k}\left|T_{v}\right|^{k}\left\{\frac{1}{Q_{n-1}} \sum_{v=1}^{n-1} q_{v}\right\}^{k-1} \\
& \leq 0(1) \sum_{v=1}^{m}\left(\frac{Q_{v}}{q_{v}}\right)^{k} q_{v}\left|\epsilon_{v}\right|^{k} \beta_{v}^{1-k}\left|T_{v}\right|^{k} \sum_{n=v+1}^{m+1} \alpha_{n}^{k-1}\left(\frac{q_{n}}{Q_{n}}\right)^{k} Q_{n-1}^{-1} \\
& \leq 0(1) \sum_{v=1}^{m}\left(\frac{\alpha_{v}}{\beta_{v}}\right)^{k-1}\left|\epsilon_{v}\right|^{k}\left|T_{v}\right|^{k}
\end{aligned}
$$

$$
\begin{aligned}
\sum_{n=2}^{m+1} \alpha_{n}^{k-1}\left|T_{n, 3}\right|^{k} & =\sum_{n=2}^{m+1} \alpha_{n}^{k-1}\left(\frac{q_{n}}{Q_{n} Q_{n-1}}\right)^{k}\left|\sum_{v=1}^{n-1}-\frac{P_{v-1}}{p_{v}} \frac{Q_{v}}{q_{v}} q_{v} \Delta \epsilon_{v} \beta_{v}^{1 / k-1} T_{v}\right|^{k} \\
& \leq \sum_{n=2}^{m+1} \alpha_{n}^{k-1}\left(\frac{q_{n}}{Q_{n}}\right)^{k} Q_{n-1}^{-1} \sum_{v=1}^{n-1}\left(\frac{P_{v-1}}{p_{v}}\right)^{k}\left(\frac{Q_{v}}{q_{v}}\right)^{k} q_{v}\left|\Delta \epsilon_{v}\right|^{k} \beta_{v}^{1-k}\left|T_{v}\right|^{k}\left\{\frac{1}{Q_{n-1}} \sum_{v=1}^{n-1} q_{v}\right\}^{k-1} \\
& \leq 0(1) \sum_{v=1}^{m}\left(\frac{P_{v-1}}{p_{v}}\right)^{k}\left(\frac{Q_{v}}{q_{v}}\right)^{k} q_{v}\left|\Delta \epsilon_{v}\right|^{k} \beta_{v}^{1-k}\left|T_{v}\right|^{k} \sum_{n=v+1}^{m+1} \alpha_{n}^{k-1}\left(\frac{q_{n}}{Q_{n}}\right)^{k} Q_{n-1}^{-1} \\
& \leq 0(1) \sum_{v=1}^{m}\left(\frac{\alpha_{v}}{\beta_{v}}\right)^{k-1}\left(\frac{P_{v-1}}{p_{v}}\right)^{k}\left|\Delta \epsilon_{v}\right|^{k}\left|T_{v}\right|^{k} .
\end{aligned}
$$

$$
\begin{aligned}
\sum_{n=1}^{m} \alpha_{n}^{k-1}\left|T_{n, 4}\right|^{k} & =\sum_{n=1}^{m} \alpha_{n}^{k-1}\left|\left(\frac{P_{n}}{p_{n}}\right)\left(\frac{q_{n}}{Q_{n}}\right) \epsilon_{n} \beta_{n}^{1 / k-1} T_{n}\right|^{k} \\
& \leq 0(1) \sum_{n=1}^{m}\left(\frac{\alpha_{n}}{\beta_{n}}\right)^{k-1}\left(\frac{P_{n}}{p_{n}}\right)^{k}\left(\frac{q_{n}}{Q_{n}}\right)^{k}\left|\epsilon_{n}\right|^{k}\left|T_{n}\right|^{k}
\end{aligned}
$$

\section{APPLICATIONS}

THEOREM 2. If

$$
\alpha_{n}=0\left(\beta_{n}\right), \quad P_{n} q_{n}=0\left(p_{n} Q_{n}\right),
$$

and (I) is satisfied, then the series $\Sigma a_{n}$ is summable $\left|\bar{N}, q_{n}, \alpha_{n}\right|_{k}$, whenever it is summable $\left|\bar{N}, p_{n}, \beta_{n}\right|_{k}$, $k \geq 1$, (that is $\left|\bar{N}, p_{n}, \beta_{n}\right|_{k} \Rightarrow\left|\bar{N}, q_{n}, \alpha_{n}\right|_{k}$ ).

PROOF. Follows from Theorem 1 by putting $\epsilon_{n}=1$.

COROLLARY 1 (Bor [1] and [4]). If 


$$
n q_{n}=0\left(Q_{n}\right), \quad Q_{n}=0\left(n q_{n}\right),
$$

then the series $\Sigma a_{n}$ is summable $\left|\bar{N}, q_{n}\right|_{k}$ iff it is summable $|C, 1|_{k}, k \geq 1$

PROOF. Applying Theorem 2 with $\alpha_{n}=Q_{n} / q_{n}, \beta_{n}=n$, and $p_{n}=1$. We have $\alpha_{n}=0\left(\beta_{n}\right)$, $P_{n} q_{n}=0\left(p_{n} Q_{n}\right)$, and (I) is satisfied. Therefore $|C, 1|_{k} \Rightarrow\left|\bar{N}, q_{n}\right|_{k}$. Now the same application of Theorem 2 with $\alpha_{n}=n, \beta_{n}=P_{n} / p_{n}$, we obtain the other way round.

COROLLARY 2 (Bor and Thorpe [5]). If

$$
P_{n} q_{n}=0\left(p_{n} Q_{n}\right), \quad p_{n} Q_{n}=0\left(P_{n} q_{n}\right)
$$

then the series $\Sigma a_{n}$ is summable $\left|\bar{N}, q_{n}\right|_{k}$ iff it is summable $\left|\bar{N}, p_{n}\right|_{k}, k \geq 1$.

PROOF. Applying Theorem 2 with $\alpha_{n}=Q_{n} / q_{n}, \beta_{n}=P_{n} / p_{n}$. Clearly $\alpha_{n}=0\left(\beta_{n}\right)$ and (I) is satisfied. Therefore $\left|\bar{N}, p_{n}\right|_{k} \Rightarrow\left|\bar{N}, q_{n}\right|_{k}$. The result is still valid if we interchange $\left\{p_{n}\right\}$ and $\left\{q_{n}\right\}$

COROLLARY 3. Suppose that (I) is satisfied for $p$ and $q$, (II) is also satisfied and that $\left\{q_{n} / p_{n}\right\}$ is nonincreasing, then the series $\Sigma a_{n}$ is summable $\left|R, q_{n}\right|_{k}$ iff it is summable $\left|R, p_{n}\right|_{k}, k \geq 1$.

PROOF. Applying Theorem 2 with $\alpha_{n}=\beta_{n}=n$. It is clear that $\left|R, p_{n}\right|_{n} \Rightarrow\left|R, q_{n}\right|_{k}$. For the other direction, it needs to be shown that (I) is satisfied if we are replacing $q_{n}$ by $p_{n}$ Since $\left\{q_{n} / p_{n}\right\}$ is nonincreasing, we have, using (II),

$$
\begin{aligned}
\sum_{n=v+1}^{\infty} \frac{n^{k-1} p_{n}^{k}}{P_{n}^{k} P_{n-1}} & =0(1) \sum_{n=v+1}^{\infty} n^{k-1}\left(\frac{q_{n}}{Q_{n}}\right)^{k} Q_{n-1}^{-1}\left(\frac{q_{n-1}}{p_{n-1}}\right) \\
& =0(1)\left(\frac{q_{v}}{p_{v}}\right) \sum_{n=v+1}^{\infty} \frac{n^{k-1} q_{n}^{k}}{Q_{n}^{k} Q_{n-1}} \\
& =0(1)\left(\frac{q_{v}}{p_{v}}\right) \frac{v^{k-1} q_{v}^{k-1}}{Q_{v}^{k}}=0\left\{\frac{v^{k-1} p_{v}^{k-1}}{P_{v}^{k}}\right\}
\end{aligned}
$$

It may be mentioned that Corollary 3 gives an alternative proof to the sufficiency part of the theorem in [2].

\section{REFERENCES}

[1] BOR, H., On two summability methods, Math. Proc. Cambridge Philos. Soc. 97 (1985), 147-149

[2] BOR, H., On the relative strength of two absolute summability methods, Proc. Amer. Math. Soc. 113 (1991), 1009-1012.

[3] SUlaiman, W T., On some summability factors of infinite series, Proc. Amer. Math. Soc. 115 (1992), 313-317.

[4] BOR, H., A note on two summability methods, Proc. Amer. Math. Soc. 98 (1986), 81-84.

[5] BOR, H. and THORPE, B., On some absolute summability methods, Analysis 7 (1987), 145-152 


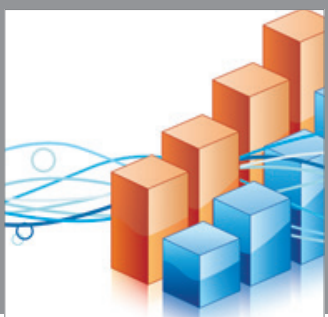

Advances in

Operations Research

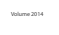

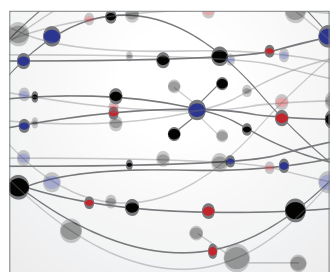

\section{The Scientific} World Journal
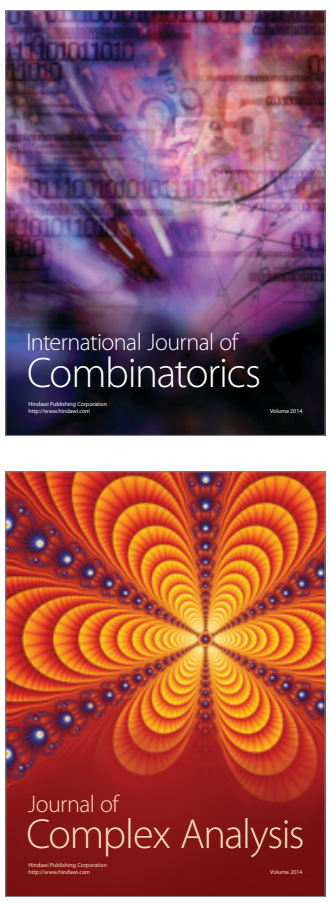

International Journal of

Mathematics and

Mathematical

Sciences
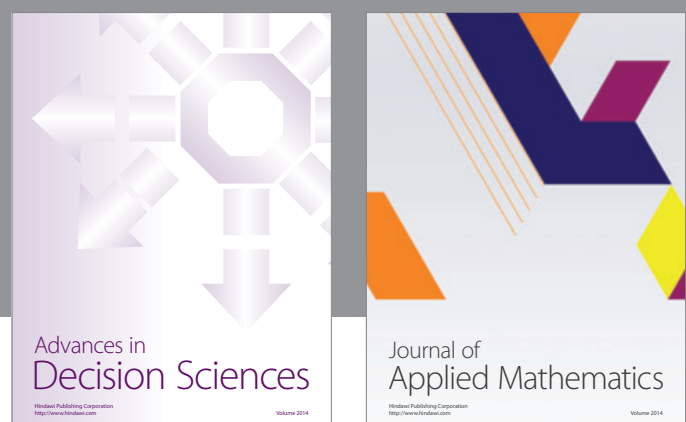

Journal of

Applied Mathematics
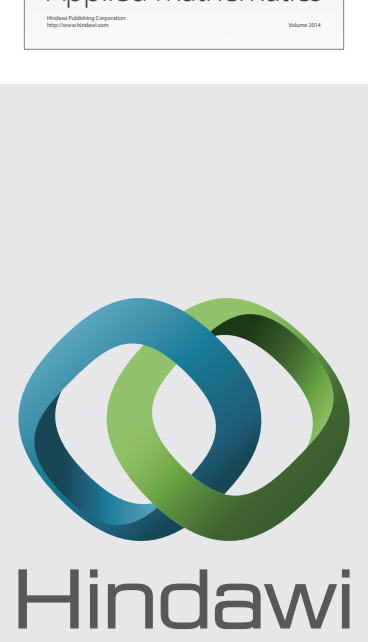

Submit your manuscripts at http://www.hindawi.com
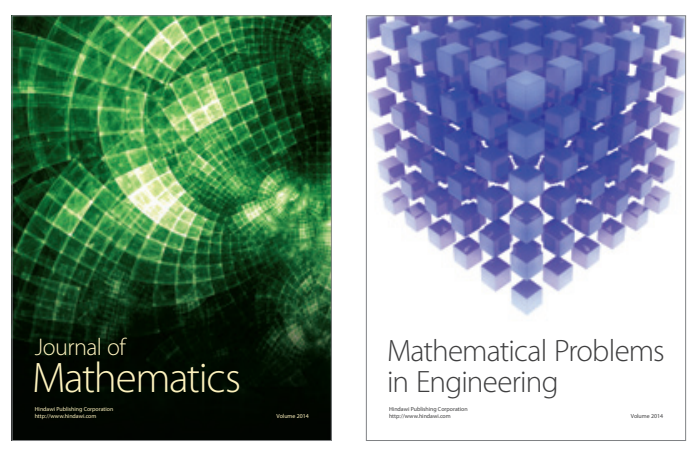

Mathematical Problems in Engineering
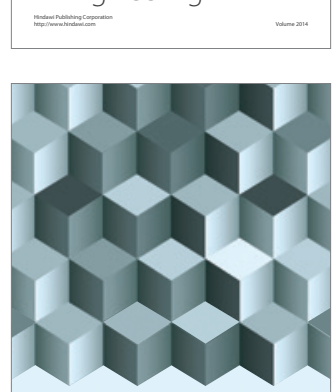

Journal of

Function Spaces
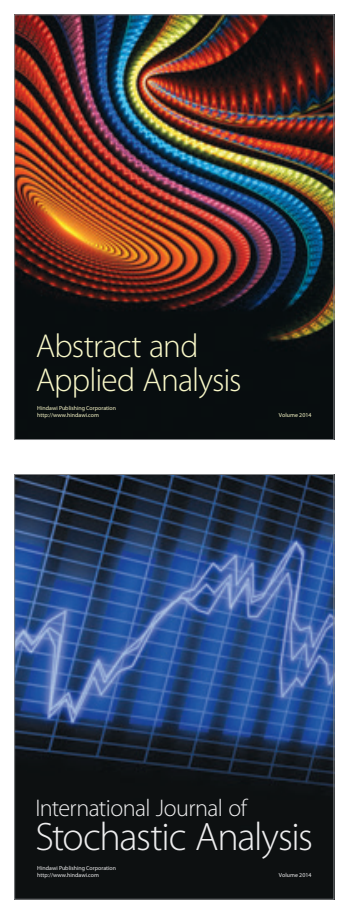

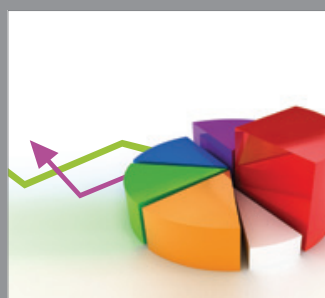

ournal of

Probability and Statistics

Promensencen
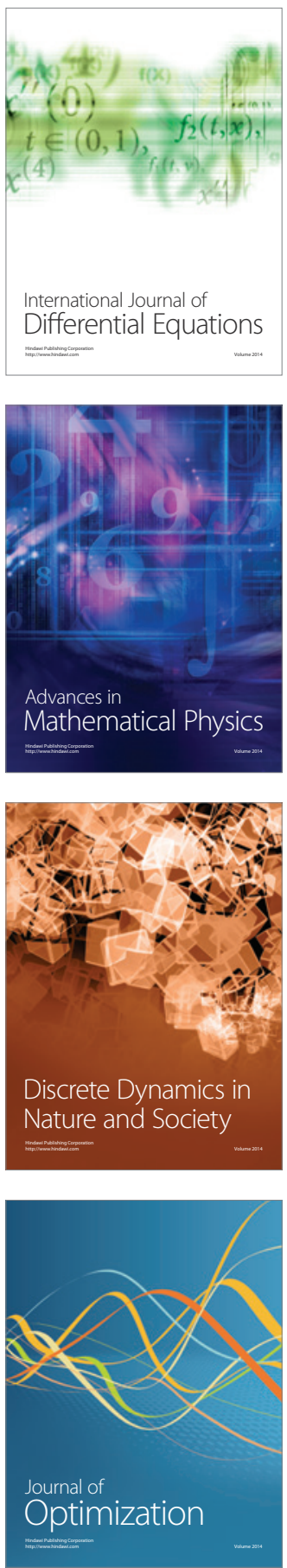